\title{
Concepção de um Mecanismo Social para o Acompanhamento Formativo entre os Contextos Computacionais: LMS e PLE
}

\author{
Ivanildo José de Melo Filho ${ }^{1,2,3}$, Rosângela Saraiva Carvalho ${ }^{2,3}$, Alex Sandro \\ Gomes $^{2,3}$, Ana Luiza de Souza Rolim ${ }^{1,2,3}$ \\ ${ }^{1}$ Instituto Federal de Pernambuco - Campus Belo Jardim (IFPE) \\ Av. Sebastião Rodrigues da Costa, S/N - CEP: 55.155-730 - Belo Jardim - PE - Brazil \\ ${ }^{2}$ Centro de Informática - Universidade Federal de Pernambuco (UFPE) \\ ${ }^{3}$ Grupo de Pesquisa Ciências Cognitivas e Tecnologia Educacional (CCTE) \\ Caixa Postal 7.851 - 54.740-540 - Recife - PE - Brazil \\ \{ivanildo.melo, analuiza.rolim\} abelojardim.ifpe.edu.br; \\ $\{$ asg, rsc5\} @cin.ufpe.br
}

\begin{abstract}
This work has the objective to present the early results which refers to the conception of a "social mechanism" that allows to interoperate computing contexts, such as LMS and PLE, in order to provide an adequate support of the formative accompaniment to the learning users. As from the review of the literature in kindred areas, we identified the requirements and it was conceived a conceptual framework to this social mechanism. The result indicates a compound framework which consists of four steps, and its conception was originated by the literature which refers to the characteristics and limitations in between these contexts.
\end{abstract}

Resumo. Esta pesquisa tem como objetivo apresentar os resultados iniciais referentes a concepção de um "mecanismo social" que permita interoperar contextos computacionais dos tipos LMS e PLE de modo a fornecer um suporte adequado ao acompanhamento formativo aos usuários aprendizes. A partir da revisão da literatura em áreas afins identificamos os requisitos e concebemos um modelo conceitual para esse mecanismo social. $O$ resultado aponta para um modelo composto por quatro estágios e sua concepção foi originada através da literatura referente às características e limitações entre esses contextos.

\section{Introdução}

É destacado por [Tavares, 2013] que as pessoas aprendem de forma autônoma com o auxílio das tecnologias. Estes leem em artigos de jornais, jogam, navegam na Internet, interagem em redes sociais, consultam sítios e criam oportunidades de aprendizagem que desafiam as instituições educacionais. $O$ autor enfatiza que estas continuam aprendendo dentro e fora da escola e opinam sobre o que querem aprender, quando e como querem aprender. E, ainda complementa que esses esforços fazem emergir novas formas de aprender que estão presentes no cotidiano das pessoas.

Entretanto, [Conde, García-Peñalvo, Casany e Forment, 2012] evidencia que 5 pontos precisam ser observados quando se faz inserção de tecnologias nos processos de 
aprendizado, são eles: a improvisação de ferramentas, os problemas relacionados à tecnologia, as questões pedagógicas, a avaliação dos aprendizes e os problemas dos Learning Management Systems (LMS) em interoperar com outras tecnologias.

Primeiro, o autor concorda [Suárez, 2008] e [SCOPEO, 2009] e alerta sobre a improvisação das ferramentas que serão utilizadas e a personalização da aprendizagem centrada nos aprendizes sugere uma ideia de que estas ações não devem ser planejadas. Tanto [Suárez, 2008] como [Conde et al., 2013] reforçam que estas ações devem ser estimadas, validadas e avaliadas de acordo com critérios diferentes. $\mathrm{O}$ segundo ponto está relacionado à tecnologia. Apesar dos grandes avanços na oferta de acesso a Web ter aumentado significativamente, o autor destaca que ainda existem problemas relacionados à qualidade do serviço que é oferecido. O terceiro ponto está diretamente ligado as questões pedagógicas; ações que auxiliem o aprendiz na sua construção da aprendizagem. O principal desafio vai ao encontro do que os modelos pedagógicos defendem quando as tecnologias são introduzidas. $\mathrm{O}$ quarto ponto está associado à avaliação dos aprendizes, [Conde et al., 2012] e [Conde, García-Peñalvo, Casany e Forment, 2013] reforçam que este processo avaliativo não é completamente formativo por não considerar todos os elementos fazem deste. Por fim, o quinto ponto está relacionado as características dos LMS (Learning Management Systems). O autor enfatiza a ausência de apoio desses ambientes para a utilização integrada de outras ferramentas. Suas características de concepção os tornam muitos complexos para interoperar com outras tecnologias.

Esses cinco pontos nos permite perceber a importância do alinhamento dos mesmos para que as ações direcionadas a formação de aprendizes sejam efetivas. $\mathrm{O}$ terceiro, quarto e quinto pontos sugerem a inexistência ou dificuldade de avaliar formativamente os aprendizes devido à ausência de um acompanhamento adequado de suas atividades. Essas dificuldades ocorrem por limitações dos LMS ou dos ambientes utilizados para personalizar a aprendizagem. Quando observados do ponto de vista dos usuários, esses ambientes são qualificados como PLE (Personal Leraning Environments). Segundo EDUCASE (2009), os LMS são centrados na estruturação dos materiais de um curso enquanto PLE são centrados nas pessoas. Os PLE podem interseccionar com funções de LMS institucional. Isso ocorre quando os aprendizes podem integrar com componentes de um LMS com os ambientes que eles utilizam.

Esta pesquisa tem como objetivo apresentar o andamento da concepção de um "mecanismo social" que interopere entre contextos computacionais dos tipos LMS e PLE. O foco desse design é o de criar um mecanismo que permita o acompanhamento formativo de atividades de aprendizagem. O "mecanismo social" proposto surgiu a partir de evidentes limitações de os LMS interoperar com outras tecnologias que medeiem o aprendizado autônomo. A literatura da área sinaliza a estabilidade da evolução dos LMS para uso acadêmico. Entretanto, expõe suas limitações, em particular com relação a funções que permitam o acompanhamento formativo de atividades individuais dos aprendizes.

As seções que compõe esta pesquisa estão organizadas da seguinte forma: a seção II apresenta a revisão de literatura sobre os Learning Management Systems (LMS), os Personal Learning Environments (PLE) $\mathrm{e}$ as possibilidades de interoperabilidade entre esses contextos educacionais. Na sequencia, a seção III traz as perspectivas desta pesquisa e introduz o conceito mecanismo social como elemento 
intermediário de integração entre estes contextos educacionais. E, por fim, a seção IV traz as considerações finais deste trabalho.

\section{Revisão da Literatura}

\subsection{Limitações dos Learning Management Systems}

Os autores [Salimah e Lim, 2013] destacam que os ambientes de aprendizagem desempenham um papel crucial na criação de experiências de aprendizagem, principalmente para estimular a autonomia e a autorregulação dos aprendizes. Nesta direção, uma das mais representativas ferramentas são os LMS [Conde et al. 2013]. Para [Sclater, 2008] os LMS são ferramentas plenamente estabelecidas e devem permanecer no campo de aprendizagem. Isto quer dizer que, seja em qualquer modalidade de ensino, esta ferramenta se encontra completamente consolidada no âmbito educacional.

Os LMS começaram a surgir há cerca de vinte anos, tendo em vista o uso generalizado da Web [Milligan, Beauvoir, Johnson, Sharples, Wilson e Liber, 2006]. Entretanto, os LMS são considerados uma tecnologia conservadora, isto se deve ao fato de que mesmos são idealizados para serem uma solução para um conjunto de problemas de ordem institucional. Sejam no gerenciamento dos aprendizes, seja no provimento de ferramentas ou entrega de conteúdos. Sob este ponto de vista, os LMS servem eficientemente as necessidades das instituições, por outro lado, de acordo com [Milligan et al., 2006] eles são frequentemente mal adaptados às necessidades dos aprendizes. É indispensável refletir, evidencia [Conde et al., 2013] que o E-Learning não termina ou está confinado a um LMS. Existe uma infinidade de ferramentas que podem ser utilizadas para complementá-lo e melhorá-lo. Inclusive, de acordo com [O'Reilly, 2005] novas aplicações decorrentes de Web 2.0 devem ser consideradas.

Apesar da sua larga utilização, [Brown e Adler, 2008] destacam que os mesmos não tem conseguido atender às necessidades que emergem das práticas de aprendizagem. Para [Conde et al., 2013], as razões fundamentais para este panorama observa que: (i) a aprendizagem deveria ser focada no aprendiz e não na instituição ou no curso [Attwell, 2007.a], (ii) existe a necessidade de fornecer suporte aos aprendizes e dar suporte à aprendizagem ao longo vida [Attwell, 2007.b], (iii) É essencial considerar a aprendizagem informal e o suporte as ferramentas Web 2.0 que promovem este modelo de aprendizado [Ajjan e Hartshorne, 2008], (iv) Os sistemas de aprendizagem devem ser capazes de evoluir com novas tecnologias [Mott e Wiley, 2009].

Diante deste cenário, os autores [Wilson, Liber, Johnson, Beauvior, Sharples e Milligan, 2007] expõem que é preciso compreender a necessidade de evoluir os LMS para sua integração com outros contextos educacionais de modo a incluir novas tendências tecnológicas, visando fornecer características sociais aos envolvidos e, acima de tudo, ser centrado nos mesmos.

\subsection{A perspectiva Personal Learning Enviroments}

Até 2006, a definição para o termo PLE permanecia indefinida [Lubensky, 2006]. O autor aponta que a concepção sobre o que deve constituir um PLE depende do ponto de vista que quem faz seu uso. Isto sugere que as prioridades para um PLE propõem-se em ser diferentes para um estudante do ensino superior, de nível técnico, para um 
coordenador de universidade, para um professor, para um tutor, para um trabalho específico, ou ainda para um indivíduo que procura um caminho alternativo de aprendizagem ao longo da sua vida.

Segundo [Attwell (a), 2007] e [Attwell (b), 2007] a ideia conceitual presente em um PLE está associada ao reconhecimento de que a aprendizagem é contínua e procura fornecer ferramentas para apoiá-la. Esta também é integrada à importância do papel do indivíduo na organização da sua própria aprendizagem. Enquanto que para [Schaffert e Hilzensauer, 2008] o conceito de PLE concentra-se em aprendizes ativos que são responsáveis e têm a oportunidade de organizar seu próprio ambiente de aprendizagem.

As tecnologias individuais que personificam o espírito dos PLE têm surgido nos últimos anos. Estas permitem e facilitam o compartilhamento de recursos através dos mais diversos meios. Nos PLE os aprendizes, de acordo com [Conde et al., 2013] [Attwell, 2007.a]; [Attwell, 2007.b]; [Schaffert e Hilzensauer, 2008] podem utilizar desde uma simples a um conjunto de ferramentas, elas podem ser customizadas para suas próprias necessidades e preferências em um único ambiente.

Muitas soluções PLE nas mais diversas áreas têm sido concebidas para o apoio a aprendizagem, entretanto, a grande maioria estão focadas em prover independência aos aprendizes, sem manter nenhuma relação ao acompanhamento de suas atividades fora dos LMS. Ao se referir aos PLE, está se tratando em fornecer autonomia aos indivíduos que fazem seu uso. Para [Mattar, 2012] um PLE é coordenado pelo próprio aluno e, de acordo com [Mota, 2009] a noção relacionada aos PLE significa o convergir de muitos dos aspectos referentes às mudanças sociais e culturais que vêm sendo provocadas pelo desenvolvimento tecnológico que decisivamente possui um forte impacto na educação e na concepção da aprendizagem.

Os autores [Mattar, 2012] e [Mota, 2009] assinalam ainda que, com o desenvolvimento das tecnologias, os alunos podem selecionar e organizar seu próprio ambiente de aprendizagem, optando pelas plataformas, ferramentas e conteúdos que são mais interessantes e que estejam em sintonia com seu estilo de aprendizagem. Além disso, [Mattar, 2012] complementa as colocações de [Downes, 2005]; [Olivier e Liber, 2001] afirmando que os LMS são ambientes centralizado nas pelas instituições. E, adicionalmente, [Valtoten, Hacklin, Dillon, Vesisenabo, Kukkonen e Hietanen, 2012] apontam que os PLE estão sendo proclamados como o próximo passo dos LMS.

\subsection{Interoperabilidade dos Contextos computacionais LMS e PLE}

As limitações dos LMS vêm sendo discutidas desde 2001. Olivier e Liber [2001] afirmam que arquiteturas baseadas em LMS não atende completamente às necessidades de aprendizagem dos alunos ao longo da vida, impedindo-os de gerir sua própria aprendizagem. Além disso, estas arquiteturas são inadequadas em fornecer aos aprendizes a continuidade, mesmo que temporária, quando esses encontram-se desconectados desses ambientes.

Estas dificuldades ou inadequações estão relacionadas a: Interoperabilidade - Os LMS possuem dificuldades para incluir padrões de interoperabilidade [Sclater, 2008]. Integração de atividades - A integração das atividades de formação entre LMS e PLE não são adequadas, visto que os PLE são concebidos para representação, classificação e acompanhamento em outras plataformas [Palmer, Sire, Bogdanov, Gillet e Wild, 2009]. Rastreabilidade - Dificuldade na rastreabilidade das atividades do usuário nos PLE e, 
portanto, gera problemas nas atividades consideradas no ambiente formal [Wilson, Sharples, Griffiths e Popat, 2009]. Execução de single-sign-on - A dificuldade de estabelecer um único ponto de entrada entre os sistemas envolvidos. [Severance, Hardin e Whyte, 2008]. E, por fim, Segurança - Descrição dificuldades em garantir a segurança da informação devido ausência da interoperabilidade. [Casquero, Portilio, Ovelar, Benito, Romo, 2010].

Contudo, diante da diversidade de recursos, soluções e dispositivos existentes, as possibilidade de integração entre PLE e LMS representam uma alternativa de solução e é um desafio atual da área. Para [Bogdanov et al., 2012] trazer flexibilidade e extensibilidade para os LMS é crucial, isto porque fornece aos professores e aprendizes uma livre escolha de tecnologias e materiais educativos que desejam usar para seus cursos. De acordo com [Conde et al., 2013] existem várias iniciativas nessa direção. No entanto, nenhuma deles está fornecendo métodos eficientes para garantir a completa integração e interação entre os LMS e PLE. Neste sentido (Wilson et al., 2007) propuseram 03 (três) cenários para esta coexistência: Cenário 01: Existência paralela dos LMS e PLE. Sendo o PLE um tipo de concepção dominante em espaços de aprendizagem informal ou em aprendizagem baseadas em competências. Enquanto que o LMS permaneceria como a tecnologia chave dos sistemas de ensino formal. Cenário 02: Os LMS disponibilizariam suas estruturas, estabelecendo um meio de interoperabilidade com os PLE. Cenário 03: Agregar as características dos PLE aos LMS, permitindo assim, incorporar o poder transformador existente nos PLE.

Baseado nas proposições de [Wilson et al., 2007] e [Conde et al., 2013] evidencia que o Cenário 01 não considera a integração, apenas a convivência simultânea. O Cenário 02 se refere à abertura dos LMS através de Web Services e iniciativas de interoperabilidade tais como: iniciativas baseadas no iGoogle, redes sociais conectadas aos LMS, possibilidade do LMS oferecer suporte a implementações de especificações de interoperabilidade, a exemplo do padrão IMS, os PLE poderiam ser concebidos com protocolo específico de comunicação [Van Hermelen, 2006] ou que a integração possa ser baseada em SOA (Service Oriented Architecture). Por fim, o Cenário 03 [Conde et al., 2013] ressalta que este considera a integração de ferramentas externas dentro do LMS. Desta maneira os aprendizes não poderiam decidir quais ferramentas seriam utilizadas, sendo eles limitados a decisões institucionais.

A interoperabilidade entre LMS institucionais e PLE aparece como uma solução para evitar que estes entre em desuso repentinamente, afirma [Melo Filho, Gomes, Carvalho, 2014]. Neste sentido, tecnologias de integração podem prover a coexistência entre estes dois contextos educacionais. Para tanto, é preciso levar em consideração que os LMS usem ou implementem esta abordagem. Do mesmo modo que os PLE tornemse instrumentos capazes de sondar e coletar informações necessárias ao acompanhamento formativo dos aprendizes. É importante observar que as pesquisas [Sclater, 2008]; [Bogdanov et al., 2012]; [Conde et al., 2012] e [Conde et al., 2013] relacionadas com a integração desses contextos educacionais têm sido apresentadas soluções e discutidas formas de estender funcionalidades dos LMS aos PLE. Entretanto, não é possível identificar uma abordagem direta relacionada ao acompanhamento formativo de atividades dos aprendizes nesse cenário. De acordo com [Melo Filho, Gomes e Carvalho, 2014]; [Melo Filho, Carvalho, Tavares e Gomes 2014] e [Melo Filho, Carvalho, Gomes e Tavares, 2014] entende-se por acompanhamento formativo a possibilidade em que o professor ou tutor possa dispor de informações sobre seus 
aprendizes referentes às atividades realizadas fora dos LMS de modo à auxiliá-los e orientá-los.

\section{Proposição de Mecanismo Social para o Acompanhamento Formativo no E-learning}

A noção de "Mecanismo Social" tem origem nas Ciências Sociais. De acordo com [Gross, 2009], trata-se uma estrutura ou processo que por algum acontecimento ou mudança de uma ou mais variáveis de entrada, faz com que uma mudança no estado de variável ou evento de saída ocorra. O autor destaca que um mecanismo social é um instrumento que media a relação de causa e efeito em um processo.

De acordo com [Bloom; Hastings e Madaus, 1983], a avaliação formativa ocorre durante todo o processo de ensino-aprendizagem, e visa identificar: o quê e quanto o discente dominou de um determinado conteúdo e o quanto e o que não foi dominado. Para [Black e William, 2009] este fenômeno ocorre na medida em que a evidência sobre o desempenho do aluno é estimulada, interpretada e utilizada por professores, alunos, ou seus pares, para tomar decisões sobre os próximos passos na aprendizagem. E, para [Brookhart, 2007], a avaliação formativa fornece informações sobre o processo de aprendizagem que os professores podem usar em suas decisões de ensino e os discentes podem usar para melhorar seu desempenho.

O termo "acompanhamento formativo" utilizado nesta pesquisa difere do termo "monitoring learning" encontrado na literatura. Apesar de quase sinônimas, o termo "monitoring learning" sugere a observação de um determinado fenômeno, num período de tempo com o objetivo de verificar se condições estabelecidas estão dentro dos padrões almejados [Lam, Keing, McNaught e Cheng, 2006] e [Yatian, Xiaomeng, Yufeng e Tongxiang, 2013]. O termo "acompanhamento formativo", aqui tratado, está consoante com [Melo Filho et al. (a) (b) (c), 2014] e tem como propósito trazer mais proximidade com o fenômeno a ser observado, no sentido de estar junto, de fornecer assistência, de orientar, dar direção, de personalizar este apoio durante toda a execução do processo.

O mecanismo social em forma de uma integração de um LMS com um PLE tem como propósito averiguar os fenômenos decorrentes de sua inserção entre os contextos computacionais LMS e PLE através do acompanhamento formativo. Para tanto, a idéia central do mecanismo é utilizar uma estratégia não-intrusiva para coletar e registrar informações sobre os eventos relacionados às atividades de aprendizagem dos aprendizes em seus PLE. De modo que permita aos aprendizes agirem individualmente, bem como, compartilhar experiências e conteúdos de aprendizagem, possam auxiliar e serem auxiliados por grupos em atividades. E, principalmente, possa fornecer aos professores e tutores subsídios para que o sentido do acompanhamento formativo destas atividades seja efetivo.

Neste sentido, a partir da revisão da literatura o mecanismo social para o acompanhamento formativo da aprendizagem proposto nesta pesquisa e representado pela Figura 1 - agrega em seu desenvolvimento as características conceituais de [Gross, 2009], bem como, as operacionais descritas por [Wilson et al., 2007] no "Cenário 2" quando se refere à abertura dos LMS na oferta de suporte a implementações de especificações de interoperabilidade com os PLE. O cenário representado pela Figura 1 possui três indicações a saber: 


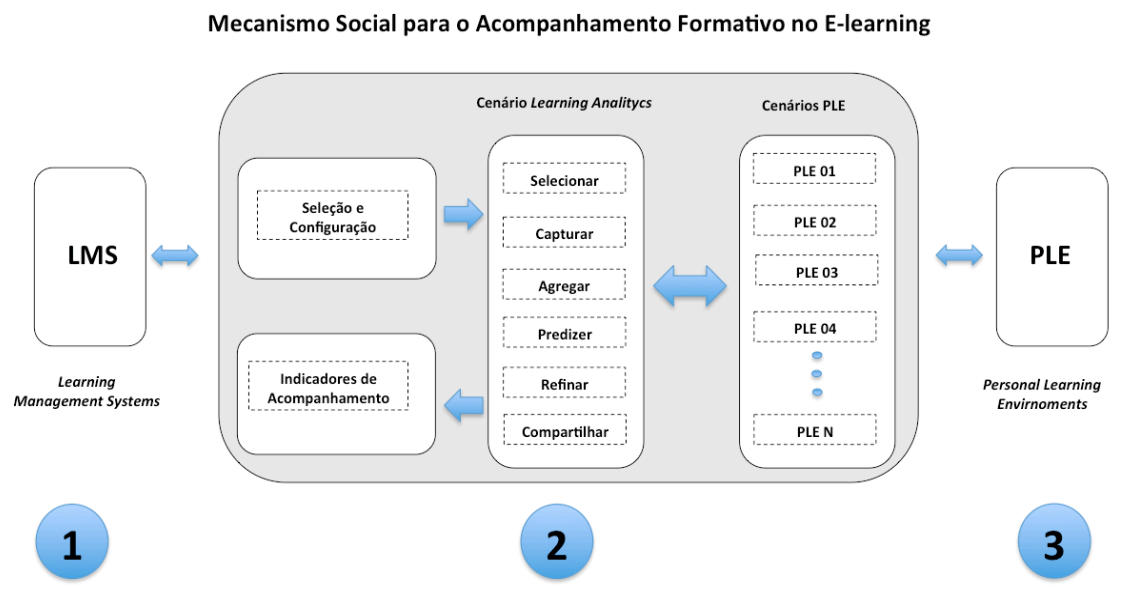

Figure 1. Mecanismo Social para o Acompanhamento Formativo no E-learning. Adaptado de [Melo Filho et. al. (b) (c), 2014].

As indicações " 1 " e "3" referem-se aos contextos educacionais PLE e LMS. A indicação " 1 " trata-se dos ambientes formais de ensino, ou também denominados de ambientes institucionais. No cenário proposto, o mecanismo social considera que uma instituição possa ter um ou mais LMS em funcionamento. A indicação "3" refere-se ao contexto no qual os PLE podem estar inseridos. Nesse caso, são representados pelos recursos da web, como também pelos mobile devices. E, a indicação "2" representa o mecanismo social para o acompanhamento formativo que é composto por 4 (quarto) estágios a saber: a) Seleção e Configuração => este estágio tem como objetivo permitir ao professor ou tutor selecionar e configurar adequadamente quais eventos relacionados às atividades dos aprendizes são relevantes serem percebidas. b) Cenário Learning Analitycs $=>$ composto por 6 (seis) elementos cujo propósito é: selecionar o cenário PLE a ser considerado, sondar e capturar as informações relacionadas as atividades dos aprendizes, agregar as informações baseadas nas definições do estágio de "Seleção e Configuração" e ser capaz de predizer possíveis futuras ações das atividades dos aprendizes, refinar as informações obtidas e compartilhar os resultados refinados. c) Cenários PLE $=>$ refere-se a definição sobre quais elementos e/ou tecnologias serão consideradas como PLE do ponto de vista do mecanismo social. d) Indicador de acompanhamento $=>$ tem como propósito fornecer de forma os indicadores de acompanhamento, baseado nas seleções prévias definidas do estágio de "Seleção e Configuração", de modo que o suporte ao acompanhamento formativo dos aprendizes possa ser diligenciado.

Os estágios que compõe o mecanismo social buscam exclusivamente averiguar os fenômenos entre os contextos educacionais, de modo que permita aos professores e/ou tutores ter subsídios ou indicadores para o acompanhamento individualizado ou em grupo. Além disso, permita perceber quem está com dificuldades no processo de aprendizagem, e por fim, que viabilize a análise em profundidade do acompanhamento formativo, permitindo que ações sejam tomadas conforme perfil e contexto de aprendizagem dos aprendizes.

\section{Considerações Finais}

Conforme descrito na introdução deste trabalho, este artigo teve como objetivo apresentar os resultados iniciais referentes a concepção de um "mecanismo social" que 
permita interoperar contextos computacionais dos tipos LMS e PLE de modo a fornecer um suporte adequado ao acompanhamento formativo aos usuários aprendizes.

Os resultados oriundos da literatura nos permite considerar que a inserção mecanismo social proposto entre estes contextos educacionais contribuirá para que o acompanhamento formativo das atividades dos aprendizes possam ser executadas pelos professores e tutores, permitindo que estes possam tomar ações adequadas ou personalizadas durante a realização de uma ou mais formações. Além disso, é preciso ressaltar que o mecanismo social proposto qualifica, por sua vez, os PLE como instrumentos colaborativos e sociais capazes de sondar e/ou captar informações relevantes ao acompanhamento formativo dos aprendizes em atividades relacionadas aos LMS ou fora deles.

Neste momento, a pesquisa está direcionada na identificação das necessidades existentes no E-learning no que tange o acompanhamento formativo de atividades para o detalhamento de cada estágio que compõe do mecanismo social proposto. Para tanto, um estudo etnográfico com professores, tutores e alunos do ensino profissional encontra-se em desenvolvimento. A partir deste estudo, serão categorizadas as necessidades identificadas e averiguadas quais estágios e seus elementos serão inseridos, modificados ou excluídos, permitindo assim, a geração dos protótipos iniciais do mecanismo.

\section{Referências}

Ajjan, H., Hartshorne, R.: Investigating faculty decisions to adopt Web 2.0 technologies: Theory and Empirical Tests. The Internet and Higher Education 11, 71-80 (2008).

Attwell (a), G.: The Personal Learning Environments - the future of eLearning? eLearning Papers 2 (2007).

Attwell (b), G.: e-Portfolios - the DNA of the Personal Learning Environment? Journal of e- Learning and Knowledge Society 3 (2007).

Black, Paul; Wiliam, Dylan. Developing the theory of formative assessment. Educational Assessment, Evaluation and Accountability (formerly: Journal of Personnel Evaluation in Education) February 2009, Volume 21, Issue 1, pp 5-31.

Bloom, Benjamin Samuel; Hastings, J. Thomas; Madaus, George F. Manual de Avaliação Formativa e Somativa do Aprendizado Escolar. São Paulo/SP: Pioneira, 1983.

Bogdanov, Evgeny; Ullrich, Carsten; Isaksson, Erik; Palmér, Matthias; Gillet, Denis. From LMS to PLE: a Step Forward through OpenSocial Apps in Moodle. Presented at: The 11th International Conference on Web-based Learning ICWL, 2012.

Brookhart, S. M. Expanding views about formative classroom assessment: A review of the literature. In J. H. McMillan (Ed.), Formative classroom assessment: Research, theory and practice. New York, NY. 2007. Teachers College Press.

Brown, J.S. \& Adler, R.P. (2008). "Minds on Fire: Open Education, the Long Tail, and Learning 2.0”. In Educause Review, January/February 2008, 43 (1), 16-32. Boulder: Educause. Retrieved January 20, 2008.

Casquero, O., Portillo, J., Ovelar, R., Benito, M., Romo, J.: iPLE Network: an integrated eLearning 2.0 architecture from University's perspective. Interactive Learning Environments Vol. 18, Iss. 3, 2010. 
Conde, M. A., García-Peñalvo, F. J., Piguillem, Jordi, Casany, M. J., Alier, M. Interoperability in eLearning contexts: interaction between LMS and PLE. A: Symposium on Languages, Applications and Technologies. "Proceedings of 1st Symposium on Languages, Applications and Technologies (SLATE 2012)". Braga: 2012.

Conde, Miguel Ángel; García-Peñalvo, Francisco José; Casany, María José; Forment, Marc Alier. Personal Learning Environments and the Integration with Learning Management Systems. Information Systems, E-learning, and Knowledge Management Research Communications in Computer and Information Science Volume 278, 2013, pp 16-21.

Downes, S. (2005). e-Learning 2.0. Retrieved September 14, 2008, from elearn. Magazine.

EDUCASE - 7 Things you should know about personal learning environments. Educase Learning Initiative. May, 2009.

Garisson,D. R. E-Learning in the 21st Century: A Framework for Research and Practice. Second Edition. 2010.

Gross, Neil. A Pragmatist Theory of Social Mechanisms. American Sociological Review, Vol. 74, No. 3 (Jun., 2009), pp. 358-379. American Sociological Associatio.

Lam, Paul; Keing, Christina, McNaught, Carmel, Cheng, Kin-Fai. Monitoring eLearning environments through analysingweb logs of institution-wide eLearning platforms. 2006. Proceedings of the 23rd annual ascilite conference: Who's learning? Whose technology?.

Lubensky, R. The present and future of Personal Learning Environments (PLE). Deliberations: Reflecting on learning and deliberating about democracy. 2006.

Mattar, J. - Tutoria e Interação em Educação a Distância - Série Educação e tecnologias - 1a. Edição - Editora Cengage Learning. São Paulo. 2012.

Melo Filho, I.J.; Gomes, A.S.; Carvalho, R.S. (a) - Acompanhamento formativo no elearning viabilizados pela integração entre Learning Management Systems e Personal Learning Environments. In: Anais do DesafIE - III Workshop de Desafios da Computação Aplicada à Educação - DesafiE2014 -. Brasília: SBC - Sociedade Brasileira de Computação, 2014. v. 01. p. 607-617.

Melo Filho, I.J.; Carvalho, R.S.; Gomes, A.S.; Tavares, E. L. C. (b) - Formative Accompaniment Border in E-Learning: Integration between LMS and PLE. In: Ireland International Conference on Education (IICE-2014), 2014, Ireland. Proceedings of Ireland International Conference on Education (IICE-2014). Dublin, 2014.

Melo Filho, I.J.; Carvalho, R.S.; Tavares, E.L.C.; Gomes, A.S. (c) - Towards the Formative Accompaniment in E-Learning: Conception of a Social Mechanism Between the Educational Contexts LMS and PLE. In: E-LEARN 2014 - World Conference on E-Learning, 2014, New Orleans. Proceedings E-LEARN 2014 World Conference on E-Learning. New Orleans, Lousiana: Association for the Advancement of Computing in Education (AACE), 2014.

Milligan, Colin D., Beauvoir, Phillip, Johnson, Mark W., Sharples, Paul, Wilson, Scott, Liber, Oleg. Developing a Reference Model to Describe the Personal Learning Environment. Innovative Approaches for Learning and Knowledge Sharing Lecture Notes in Computer Science Volume 4227, 2006, pp 506-511. 
Mota, José. Da Web 2.0 ao e-Learning 2.0: Aprender na Rede. Dissertação de Mestrado. Versão Online, Universidade Aberta. Portugal. 2009.

Mott, J., Wiley, D.: Open for Learning: The CMS and the Open Learning Network. In: Education - Exploring Our Connective Educational Landscape. University of Regina, Saskatchewan (2009).

Olivier, B. e Liber, O. "Lifelong learning: the need for portable personal learning environments and supporting interoperability standards". SSGRR 2002w International Conference on Advances in Infrastructure for Electronic Business, Education, Science and Medicine on the Internet, L'Aquilla, Italy. 2001.

O'Reilly, T.: What is Web 2.0: Design Patterns and Business Models for the Next Generation of Software (2005).

Palmér, M., Sire, S., Bogdanov, E., Gillet, D., Wild, F.: Mapping Web Personal Learning Environments. In: Wild, F., Kalz, M., Palmér, M., Muiller, D. (eds.) Second International Workshop on Mashup Personal Learning Environments (MUPPLE 2009), vol. 506, pp. 31-46. CEUR-WS.org, Nize (2009).

Schaffert, S. e Hilzensauer, W. On the way towards personal learning environments: seven crucial aspects. Elearning Papers, 9. 2008.

Sclater, Niall. "Web 2.0, Personal Learning Environments, and the Future of Learning Management Systems" (Research Bulletin, Issue 13). Boulder, CO: EDUCAUSE Center for Analysis and Research, 2008.

SCOPEO (2009). Formación Web 2.0, Monográfico SCOPEO, $\mathrm{n}^{\circ} 1$. Observatorio de la actividad, la innovación y las tendências en la Formación en Red.

Severance, C., Hardin, J., Whyte, A.: The coming functionality mash-up in Personal Learning Environments. Interactive Learning Environments 16, 47-62 (2008).

Suárez, C. Virtualidad y Educación. Bases teóricas y empíricas del aprendizaje cooperativo en Internet. 2008. Lima, Editorial de la Universidad Ricardo Palma.

Tavares, E.L.C. Interfaces da Línguística e Linguagem dos Aplicativos para Aprendizagem com Mobilidade no Âmbito do Ensino de Inglês. Dissertação de Mestrado - UNICAP - Universidade Católica de Pernambuco. Pró-Reitoria Acadêmica. Mestrado em Ciências da Linguagem, 2013.

Valtonen, T.; Hacklin, S.; Dillon, P., Vesisenaho, M.; Kukkonen J.; Hietanen A. Perspectives on personal learning environments held by vocational students, Computers \& Education, Volume 58, Issue 2, February 2012, Pages 732-739.

Van Harmelen, M. (2006). Personal Learning Environments. In: Computer Society (Eds.) Sixth IEEE International Conference on Advanced Learning Technologies (ICALT06), (pp.815-816) London, England, 02 April, 06 June, 2006. London: UK.

Wilson, S., Liber, O., Johnson, M., Beauvoir, P., Sharples, P., Milligan, C.: Personal Learning Environments: Challenging the dominant design of educational systems. Journal of e-Learning and Knowledge Society 3, 27-38 (2007).

Wilson, S., Sharples, P., Griffiths, D., Popat, K.: Moodle Wave: Reinventing the VLE using Widget technologies. In: Wild, F., Kalz, M., Palmér, M., Müller, D. (eds.) Mash-Up Personal Learning Environments - 2st Workshop MUPPLE 2009, vol. 506, pp. 47-58. CEUR Proceedings, Nize (2009).

Yatian, Chen; Xiaomeng, Lv; Yufeng, Jiang; Tongxiang, Wu, "Research on learningmonitoring system for E-Learning," Computer Science \& Education (ICCSE), 2013 8th International Conference on , vol., no., pp.16,18, 26-28 April 2013. 